\title{
Development of Information Triangulation Method for Prediction of Earthquake Center Zone Using Distributed Measurements of Emitted Electromagnetic Radiation
}

\author{
H. H. Asadov, L. I. Nuriyeva \\ Azerbaijan National Aerospace Agency, Baku, Azerbaijan \\ Email: asadzade@rambler.ru
}

How to cite this paper: Asadov, H.H. and Nuriyeva, L.I. (2020) Development of Information Triangulation Method for Prediction of Earthquake Center Zone Using Distributed Measurements of Emitted Electromagnetic Radiation. Positioning, 11, 1-9. https://doi.org/10.4236/pos.2020.111001

Received: February 11, 2020

Accepted: February 26, 2020

Published: February 29, 2020

Copyright $\odot 2020$ by author(s) and Scientific Research Publishing Inc. This work is licensed under the Creative Commons Attribution International License (CC BY 4.0).

http://creativecommons.org/licenses/by/4.0/

\begin{abstract}
The new method for prediction of earthquake center zone is suggested. The method is based on feature of amount of registered information to reach its maximum upon some condition regulating interrelation of major parameters of used distributed measuring system. The mathematical basis of suggested is based on known integrated Shannon formula of amount of information and integral limitation condition, expressing fixed position of used sensors. As a result of held researches, new method of information trangulation method for determination of earthquake center zone is suggested. The mathematical grounding and the operational algorithm of the method are given.
\end{abstract}

\section{Keywords}

Earthquake, Prediction, Sensors, Information, Optimization

\section{Introduction}

Development of methods for prediction of earthquake attracted attention of many scientists [1]-[5]. As it is noted in [6], development of simplified models to estimate expected electromagnetic radiation during earthquake process is important task. Generated during preparation of earthquake process ULF electromagnetic emission could be detected by magnetometers.

According to [7], seismic events are preceded by increase of stress deep in Earth, and as a result, by generation of ultra-low frequency electromagnetic waves. At the Earth's surface, it is resulted by ionization of air, which can be detected by animals. 
In line with [8], speed of occurence of seismological signals before earthquakes depending on volume of cracked rock at depth is a primary local source of precursor signals. As it is noted in [9], researchers on possible earthquake prediction must be carried out on a deterministic basis. That implies the necessity to carry out combined ground and space observations of earthquake precursor. Earthquake precursory phenomes include not only emission of ULF electromagnetic waves rad, but also visible and near infrared light. According to [10], during seismic event, amplitude of electromagnetic radiation increases as long as the crack continues to grow because new atomic bonds are severed.

As it is noted in [11], seismo-electromagnetic precursory based prediction method appeared in the scientific literature for many decades but motivation is given recently in [12] [13]. Well-known triangulation method based on propagation of electromagnetic waves was considered in [14] [15]. The principle of this method is based on speed of movement of electromagnetic waves ant fixation of their arrival. Graphical realization of the method based on registration of arrived wave at three stations is illustrated in Figure 1 [11].

At the same time, numerous sums of effects of rocks cracking and random character of exposures of seismical activity lead to statistical nature of forerunners of earthquakes. Particularly, as regard a normal electromagnetic irradiation, it is practically not possible to select the most informative frequencies, which should be observed. The optimal azimuth angle of receiving of such radiation usually is also unknown, which creates additional problems for optimal solution of earthquake center prediction problem. Due to the above, it can be concluded, that the system destined for gathering and processing of seismological information of electromagnetic radiation type should guarantee receipt and processing maximal amount of such information. This condition leads us to construct the single informational model of electromagnetic radiation before earthquakes, optimization of which makes it possible to predict the center of earthquakes using informational criteria. Thus the aim of this research is development of new information.

\section{Suggested Method}

\subsection{Mathematical Basics}

First of all, we should stress out that the intensity of electromagnetic radiation depends on two factors:

1) Distance between the receiver and center zone of earthquake- $L$.

2) Frequency of electromagnetic radiation $-F$.

Above dependences in both case expressed by fading of intensity of electromagnetic radiation.

Taking into account above, we propose, that each seismosensor can be characterized in the three-sized space as it is shown in Figure 1, where the point $\mathrm{A}$ characterize any single seismosensor, which located at the distance $L_{A}$ from center of earthquake, receives electromagnetic radiation of frequency $F_{A}$, and duration of receipt is $T_{A}$ (Figure 2). 


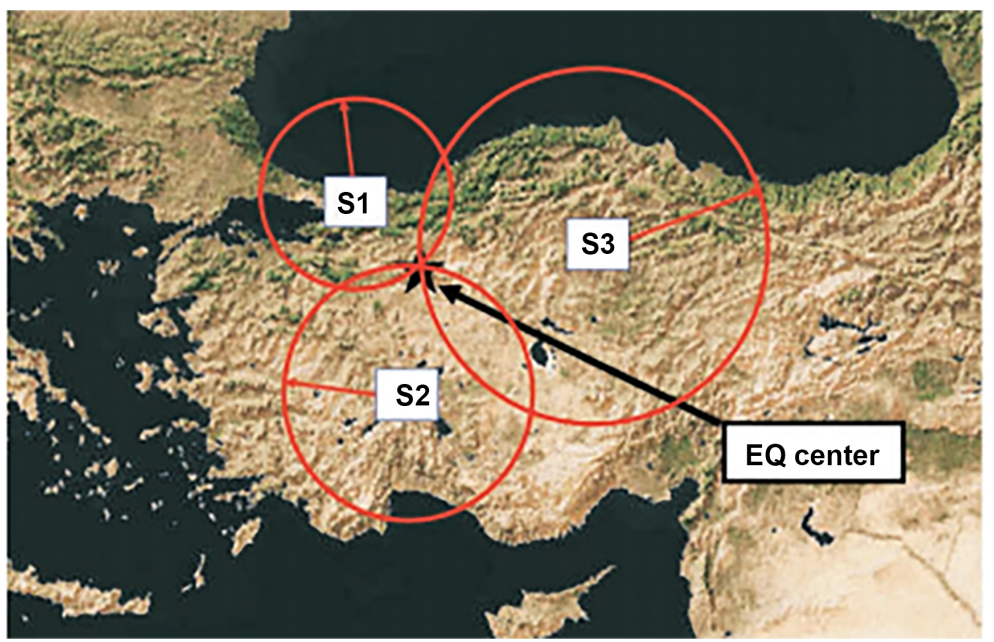

Figure 1. Scheme of triangulation method applied for determination of center of earthquake.

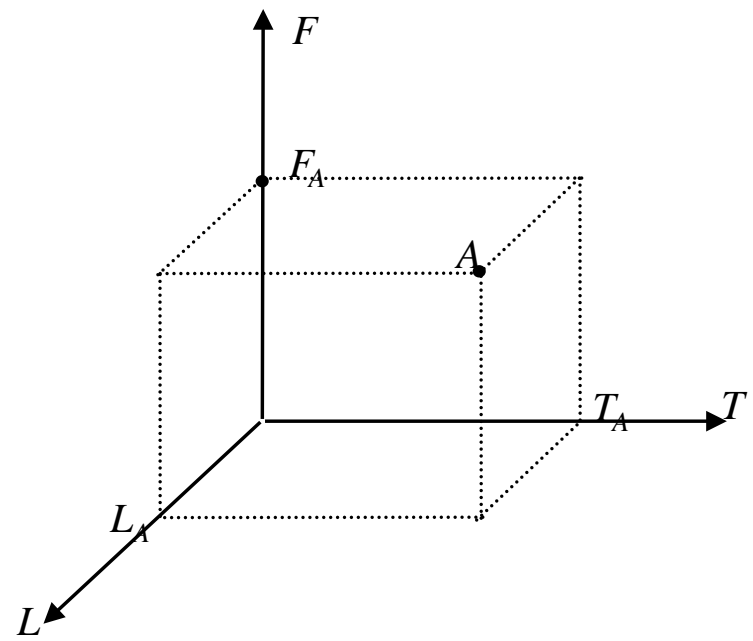

Figure 2. Three-sized interpretation on single seismosensor.

Taking into account of above, we can find the signal/noise ratio $\psi$ of the received signal as

$$
\psi=(L, F)=\psi_{0}+\frac{\partial \psi}{\partial L} \cdot d L+\frac{\partial \psi}{\partial F} \cdot d F,
$$

where $\psi_{0}=\psi\left(L=L_{0} ; F=F_{0}\right)$.

Now considering the whole net of seismosensors consisting of $n$ ones, and taking into account, that optimal system of processing is designed in such a manner, that it receives signals of duration $T_{i}, i=\overline{1, n}$, where value of $T_{i}$ increases depending on $i$. In this case, the amount of information, which is gathered from all $n$ sensors can be assessed as follows

$$
\Phi=\sum_{i=1}^{n} \frac{T_{i}}{\Delta T} \log _{2}\left[\psi_{0}+\frac{\partial \psi}{\partial L} d L+\frac{\partial \psi}{\partial F} d F+1\right] .
$$

Using formula (2), we can solve two type of optimization task, which allow us 
to form two methods for prediction of earthquakes center using static distributed set of receivers and maximal informational criteria.

1) Receive of electromagnetic radiation at the same frequency.

2) Receive of electromagnetic radiation at the same distance used for confirmation of found center of earthquake on first method.

In the first case, the ratio of signal/noise $\psi$ in the channel of propagation of Low-frequency electromagnetic radiation from the source of these signals as far as receiver in the first approximation can be found as

$$
\psi=\psi_{0}+\psi^{\prime} L
$$

where: $\psi_{0}=\psi(L=0) ; \psi^{\prime}=\frac{\mathrm{d} \psi}{\mathrm{d} L}$.

During the whole period of information retrieval, total amount of gained information can be found as

$$
M_{0}=\sum_{i=1}^{n} M_{i}=\sum_{i=1}^{n} \frac{T_{i}}{\Delta t} \log _{2}\left(\psi_{0}+\omega^{\prime} L_{i}+1\right),
$$

where $T_{i}$-duration of information retrieval from the sensor numbered as $i$.

Next, we use a limitation condition, formed as a result of fixed construction of the set of sensors, i.e.

$$
\sum_{i=1}^{n}\left(\psi_{0}+\psi^{\prime} L_{i}+1\right)=\text { const }
$$

Taking into account above, the functional of effectiveness can be formed as

$$
\Phi=\int_{0}^{T_{\max }}\left[\frac{T}{\Delta t} \log _{2}\left(\psi_{0}+\psi^{\prime} L+1\right)+\lambda\left(\psi_{0}+\psi^{\prime} L+1\right)\right] \mathrm{d} T,
$$

where $\lambda$ is L'Agrange multiplier.

According to the principle of optimal lowering of dimensionality [2], we should find such type of optimal function $L=\varphi(T)$ which would lead the functional of effectiveness to its maximal value.

Solution of the formulated maximization task using Euler's formula gives us following type of said function

$$
L=\frac{\psi_{0}+1}{\left|\psi^{\prime}\right|}-\frac{T \psi_{0}}{T_{\max }\left|\psi^{\prime}\right|} .
$$

As a result, we have the possibility to carry out an adaptive control of seismosensors, i.e. all system of gathering and processing of seismic information.

\subsection{Development of New Method}

On the basis of above result, we can propose a new method of informational for casting of epicenter of expected earthquakes. We assume that seismosensors are placed on the territory with high seismic risk (Figure 3 ).

In order to forecast the epicenter of earthquake we should designate set of values $\left\{L_{i}\right\}$, and set of values $\left\{T_{i}\right\}$, where dependence between $L_{i}$ and $T_{i}$ should 


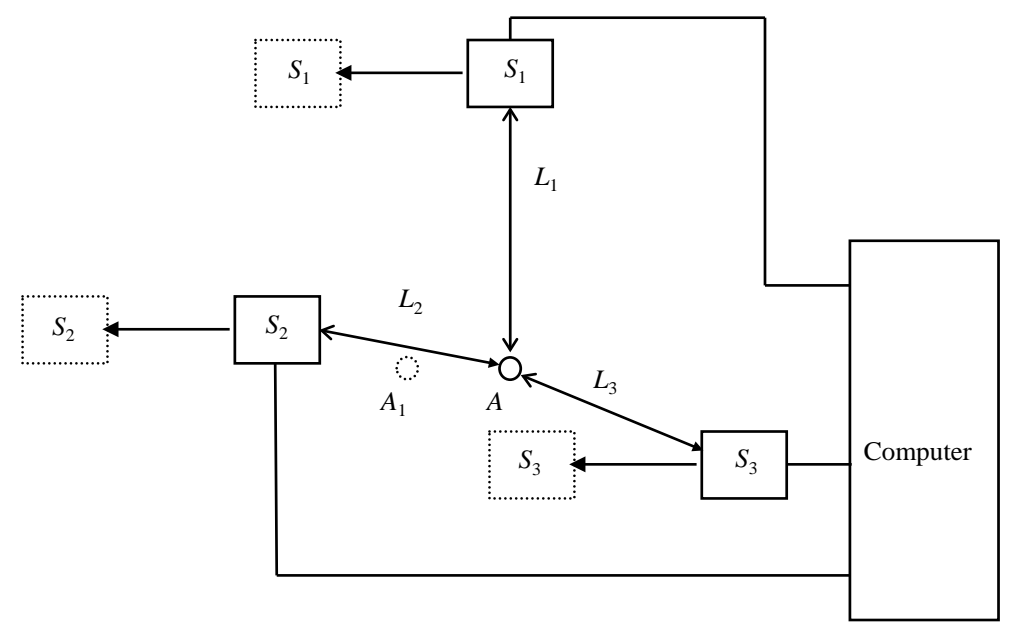

Figure 3. Explanation of method of forecasting.

be in line with formula (3), which guarantee maximum value of total information gathered from seismosensors. Selected three seismosensors $S_{1}, S_{2}, S_{3}$ will be commutated during time period $T_{i}$ determined by formula (3). Under above conditions computer should detect maximum amount of information, if the point $A$ is actually the center of expected earthquake. Selected set of sensors should be moved along the territory in order to detect maximum of gathered information. More strictly no sensors, but selector contour of three sensors should be moved over the territory via fixed set of sensors and movement of this contour should be stopped if maximal amount of information is reached. Then using known set of $L_{i}$, we can find needed placecenter of earthquake.

Receive of electromagnetic radiation at the same distance used for confirmation of found center of the earthquake on first method.

In this case, the ratio signal/noise $\psi$ in the channel of propagation of lowfrequency electromagnetic radiation from the source of these signals as far as receiver in the first approximation can be found as

$$
\psi=\psi_{0}+\psi_{F}^{\prime} \cdot F
$$

where $\psi_{F}^{\prime}=\frac{\partial \psi}{\partial F} ; F$-frequency of electromagnetic radiation.

During the whole period of information retrieval total amount of gathered information can be found as

$$
M_{0}=\sum_{i=1}^{n} M_{i}=\sum_{i=1}^{n} \frac{T_{i}}{\Delta t} \log _{2}\left(\psi_{01}+\psi_{F}^{\prime} \cdot F+1\right),
$$

where $T_{i}$-duration of information retrieval from the sensor numbered as $i$.

Then we use a limitation condition

$$
\sum_{i=1}^{n}\left(\psi_{01}+\psi_{F}^{\prime} \cdot F_{i}+1\right)=\text { const }
$$

or

$$
\sum_{i=1}^{n} F_{i}=\text { const }
$$


A limitation condition (6) means, that band of reasonably received frequencies is limited.

Taking into account of (5) and (6) we can form the functional of effectiveness as follows:

$$
\Phi=\int_{0}^{T_{\max }}\left[\frac{T}{\Delta T} \log _{2}\left(\psi_{01}+\psi_{F}^{\prime} \cdot F+1\right)+\lambda\left(\psi_{01}+\psi_{F}^{\prime} \cdot F+1\right)\right] \mathrm{d} T
$$

where

$T$-time of information retrieval; $\lambda$-multiplier of L'Agrang.

According to the principle of optimal lowering of dimensionality [16], we should find such type of optimal function $L=\phi(T)$ which would lead the functional of effectiveness (7) to its maximal value.

Solution of above optimization task using Euler's formula gives us following type of said function

$$
F=\varphi(T)=\frac{\psi_{0}+1}{\left|\psi_{F}^{\prime}\right|}-\frac{T \cdot \psi_{0}}{T_{\max }\left|\psi_{F}^{\prime}\right|}
$$

As a result, we obtain the possibility to carry out an adaptive control of seismosensors, i.e. whole system of gathering and processing of seismic information. This does mean, that sensor with the lowest frequency of received signal should be examined during uppermost time period in order to reach maximal efficiency of the system.

On the basis of above result, we can propose a new second method for informational forecasting of center of expected earthquakes. We assume that seismosensors are placed on the territory with high seismic risc, forming a rectangular Net (Figure 4).

In order to forecast the place of earthquake we should designate a set of values $\left\{F_{i}\right\} ; i=\overline{1, n}$, and set of values $\left\{T_{i}\right\} ; i=\overline{1, n}$, where dependence between $F_{i}$ and $T_{i}$ should be in line with formula (8), which guarantees reaching of maximum value of total information, gathered from seismosensors. Here we should note, that each sensor consists of $n$ receiver with fixed frequency $\left\{F_{i}\right\} ; i=\overline{1, n}$.

Hence, each sensor is compound on $n$ number of receiver and makes it possible to receive in the frequency band $\left\{F_{1}, F_{2}, \cdots, F_{n}\right\}$.

Selected contour of four seismosensors (in Figure 3 they are $S_{1}, S_{2}, S_{4}, S_{5}$ ) will be moved across the high rise territory-area of placement of sensors, and the movement of the contour should be stopped in the point, where the estimated value of functional of effectiveness reaches a maximal value. Such assessment can be realized using computer, which should also control movement of the contour of sensors.

It should be noted that the above second method is used for confirmation of place of earthquake center, determined by the first method.

On the basis of two above methods we can formulate single general algorithm for prediction of earthquake's center zone (Figure 5), which consists of following steps: 


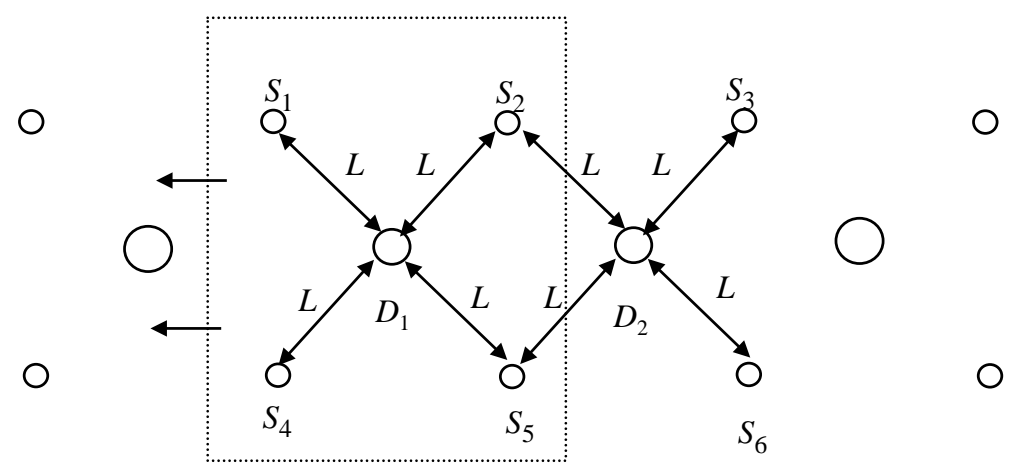

Figure 4. Movement of the contour aeross the not of sensors.

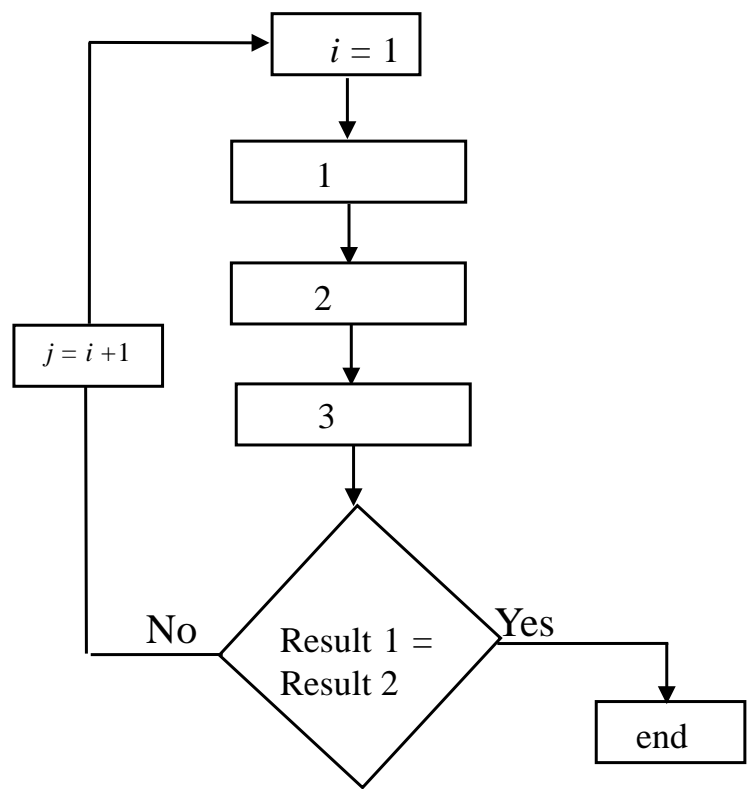

Figure 5. Chart-scheme of algorithm.

1) Using of fixed rectangular net of seismosensors for determination of center zone on first method.

2) Construction of non-rectangular mobile micro-net consisting of three or four sensors located at the same distance from found center zone.

3). Application of the second method.

4) Confirmation, that two results on points 1 and 3 are the same.

Taking into consideration of above we conclude, that a proposed new informational method of forecasting of earthquake center zone may increase effectiveness of measures taken for global forecasting of seismic events.

\section{Conclusion}

Thus, the new method for prediction of earthquake center zone is suggested. The method is based on feature of amount of registered information to reach its maximum upon some condition regulating interrelation of major parameters of used distributed measuring system. The mathematical basis of suggested method 
is based on known integrated Shannon formula of amount of information and integral limitation condition, expression fixed position of used sensors.

Now we can note major result of held research.

The new method of information triangulation method for determination of earthquake center zone is suggested.

The mathematical grounding and the operational algorithm of the method are given.

\section{Conflicts of Interest}

The authors declare no conflicts of interest regarding the publication of this paper.

\section{References}

[1] Geller, R.J. (1997) Earthquake Prediction: A Critical Review. Geophysical Journal International, 131, 425-450. https://doi.org/10.1111/j.1365-246X.1997.tb06588.x

[2] Kagan, Y.Y. and Knopoff, L. (1987) Statistical Short-Term Earthquake Prediction. Science, 236, 1563-1567. https://doi.org/10.1126/science.236.4808.1563

[3] Schall, R.B. (1988) An Evaluation of the Animal-Behavior Theory for Earthquake Prediction. California Geology, 41, 41-45.

[4] Geller, R.J. (1991) Shake-Up for Earthquake Prediction. Nature, 352, 275-276. https://doi.org/10.1038/352275a0

[5] Wyass, M. (1991) Evaluation of Proposed Earthquake Precursors. Am. Geophys. Un., Washington DC. https://doi.org/10.1029/SP032

[6] Surkov, V.V. and Pilipenko, V.A. (2014) Estimate of ULF Electromagnetic Noise Caused by a Fluid Flow during Seismic or Volcano Activity. Natural Hazards and Earth System Sciences Discussions, 2, 6475-6497. https://doi.org/10.5194/nhessd-2-6475-2014

[7] Freund, F. and Stolc, V. (2013) Nature of Pre-Earthquake Phenomena and Their Effects on Living Organisms. Animals, 3, 513-531.

https://doi.org/10.3390/ani3020513

[8] Outkin, V.I., Kozlova, I.A., Yurkov, A.K., Dutta, P.K., Mishra, O.P. and Naskar, M.K. (2013) Radon Monitoring as a Possible Indicator of Tectonic Events. Earth System Dynamics, 4, 93-107. https://doi.org/10.5194/esdd-4-93-2013

[9] Sgrigna, V. and Conti, L. (2012) A Deterministic Approach to Earthquake Prediction. International Journal of Geophysics, 2012, Article ID: 406278. https://doi.org/10.1155/2012/406278

[10] Frid, V., Rabinovitch, A. and Bahat, D. (2003) Fracture Induced Electromagnetic Radiation. Journal of physics D: Applied Physics, 36, 1620-1628. https://doi.org/10.1088/0022-3727/36/13/330

[11] Sevgi, L. (2007) A Critical Review of Electromagnetic Precursors and Earthquake Prediction. Turkish Journal of Electrical Engineering and Computer Sciences, 15, $1-15$.

[12] Bleier, T. and Freund, F. (2005) Earthquake Alarm. IEEE Spectrum, 42, 16-21. https://doi.org/10.1109/MSPEC.2005.1549778

[13] http://www.earthquakeprediction.gr/index.htm.

[14] http://quake.wr.usgs.gov/info/eqlocation/index.html. 
[15] http://quake.wr.usgs.gov/research/seismology/index.html.

[16] Asadov, H.H. (2003) The Principle of Dimensionality Lowering for Synthesis and Optimization of a Subclass of Measuring Systems with Fading Signals on Example of Remote Sensing Systems. Journal: Devices and Systems. Control, Testing, Diagnostics, No. 8, 60-67. (In Moscow) 